\title{
Development of 3D Printing Software for Medical Customized Treatments
}

\author{
Jiyoung Yoon ${ }^{l,+}$, Soowon Seo ${ }^{l, *}$, Haeyong Choi ${ }^{l}$ and Cheolhwan Kim ${ }^{2}$ \\ ${ }^{1}$ Medical Device Development Center, Daegu-Gyeongbuk Medical Innovation Foundation, \\ 80, Dongnae-ro, Dong-gu, Daegu City, 41061, Republic of Korea. \\ 23D Convergence Technology Center, Institute of Advanced Convergence Technology, \\ 70, Dongnae-ro, Dong-gu, Daegu City, 41061, Republic of Korea.
}

\begin{abstract}
D printing technology is widely used in the medical field. In particular, many customized treatments are being produced and studied. Customized treatments can be created using 3D printers, and 3D models and Gcodes for individuals can be created using tomographic images. It is important to create a 3D model by precise segmentation of tomographic images. In order to increase the precision of segmentation, we applied the adaptive thresholding method with morphological process. We applied a look-up table to quickly correct holes and vertex errors in 3D models. In this paper, we proposed the 3D printing software development method for customized treatments.
\end{abstract}

Key words: $3 D$ Printing, Tomographic Images Segmentation, Customized Treatments, 3D Model

\section{INTRODUCTION}

As 3D printing technology evolves, it is being used in many industries. In particular, many customized treatments are being produced and studied. Customized treatments are medical auxiliary products that adapts to the shape of the patient's body, such as the shape of patients' appearance, organs and bones. 3D printers are used to make personalized medical implants such as hearing aids, dentures, prosthetics and surgical guides. By creating virtual bodies for surgical simulation, it has contributed to shortening of operation time and improving surgical success rate. Virtual bodies are also used for education of researchers in the medical field. Customized treatments are used in many researches and practical treatments around the world. Fig 1 is an example of customized treatments.

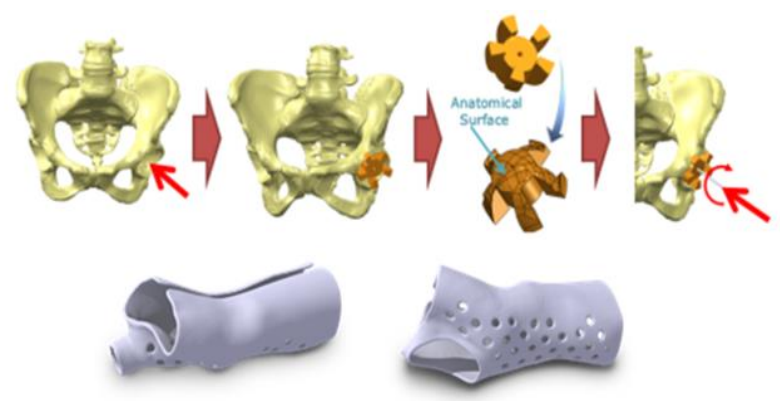

Fig 1 Customized treatments
Recently, 3D cell printing technology using inducible pluripotent stem cells has been researched to restore function of damaged tissue or organ. The global market for medical 3D printers is expected to grow from US $\$ 540$ million in 2015 to US $\$ 1.29$ billion by 2021with CAGR of $15.4 \%$ [1].

3D models and G-codes are required to create customized treatments. Customized treatments can be created using 3D printers, and 3D models and G-codes for individuals can be created using tomographic images. It is important to create $3 \mathrm{D}$ models by precise segmentation of tomographic images. Precise segmentation of tomographic images is essential to create $3 \mathrm{D}$ models that match the shape and size of human components. To create error-free 3D printing output, 3D models should be free of errors. G-codes are generated based on generated 3D models. G-code [2] is a programming language used to control 3D printers. The 3D printers are controlled by the G-Code and output products. In this paper, we proposed the 3D printing software development method for customized treatments.

\section{EXPERIMENT}

\section{Design}

We have developed the 3D model generation software and the G-code generation software for 3D printing. The 3D model generation software segments tomographic 
images and makes 3D models using segmentation results. This software corrects errors in 3D models and converts 3D models into STL files [3] to make G-code. The 3D model generation software includes the ability to visualize and to modify 3D models. The G-code generation software slices 3D models for 3D printing and creates G-codes using sliced layers. We did not develop the G-code generation software, we used Ultimaker's CuraEngine as open source. CuraEngine supports various 3D printing settings and slices the STL model to create G-code. Two software are operated by the 3D model generation software as main software and G-code generation software as auxiliary software. The 3D model generation software has GUI and executes the G-code generation software as a process when generating G-codes. Fig 2 shows the flow of the operation of two software.

\begin{tabular}{|c|c|c|}
\hline $\begin{array}{l}\text { 3D model } \\
\text { generation SW }\end{array}$ & & $\begin{array}{c}\text { G-code } \\
\text { generation SW }\end{array}$ \\
\hline \multirow{2}{*}{$\begin{array}{l}\text { - Generate } 3 D \text { model } \\
\text { and } 3 D \text { printer } \\
\text { setting data } \\
\text { - Process execution } \\
\text { request and create } \\
\text { progress monitoring } \\
\text { thread }\end{array}$} & & \\
\hline & $\begin{array}{l}\text { 1. Execution process } \\
\text { and send setting } \\
\text { data }\end{array}$ & \multirow[t]{2}{*}{$\begin{array}{l}\text { - Execute process } \\
\text { - Slicing and G-code } \\
\text { generation }\end{array}$} \\
\hline $\begin{array}{l}\text { - Generate } 3 D \text { model } \\
\text { and } 3 D \text { printer } \\
\text { setting data } \\
\text { - Process execution } \\
\text { request and create } \\
\text { progress monitoring } \\
\text { thread }\end{array}$ & $\begin{array}{l}\text { 2. Communicate } \\
\text { progress and } \\
\text { error information }\end{array}$ & \\
\hline $\begin{array}{l}\text { - Progress monitoring } \\
\text { thread termination }\end{array}$ & $\begin{array}{l}3 \text { Communicate } \\
\text { progress } \\
\text { termination }\end{array}$ & $\begin{array}{l}\text { - Generate G-Code } \\
\text { - Process termination }\end{array}$ \\
\hline
\end{tabular}

Fig 2 The flow of 3D model and G-code generation software

\section{D model generation}

The CT data were CBCT (Cone Beam Computed Tomography) images with a resolution of $512 \times 512$ pixels. In CT data, segmentation [4] is required to make a desired region into 3D models. We used the adaptive threshold method to segment CT data. The adaptive threshold method is a method to determine the region of interest by performing initial segmentation by applying a global threshold and applying the local threshold value to each pixel by applying the Otsu algorithm [5], which is a probability statistical method to the boundary pixels of the object and background. The Otsu algorithm finds the correct areas by about 50 recursive operations. Fig 3 shows the results of tooth segmentation on CT using the graph cut method and the adaptive threshold method.

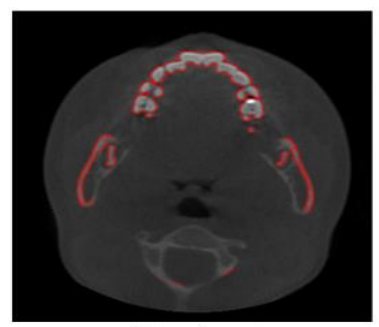

Graph cut

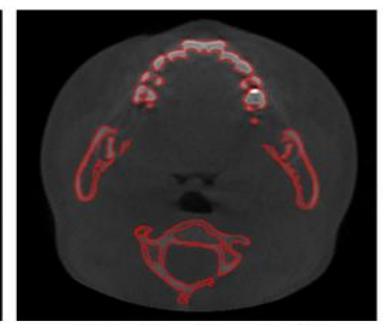

Adaptive threshold
Fig 3 The results of tooth segmentation on CT

The segmentation result of the region of interest using the adaptive threshold method finds regions with high brightness values in images, but cannot find regions with relatively small brightness values and thin shapes. To solve this problem, we have supplemented the adaptive segmentation algorithm. It divides the region of interest primarily using the adaptive threshold method and applies the morphology method to the divided regions [6]. In this method, thin-shaped areas with small brightness values are selected and made into connected objects. After finding additional areas of interest through the morphology method, adaptive threshold method is applied again to this area only. By doing this additional work, the areas which was not found when only the adaptive threshold method was applied can be found, and more accurate areas of interest are found. When using the general adaptive threshold method, Otsu algorithm classifies objects with low brightness values as backgrounds. In order to improve this problem, the adaptive threshold method is applied again, excluding the object having the strongest brightness value as much as possible. In this way, it is possible to segment objects with weak brightness values classified as backgrounds. Fig 4 shows an example of an object with high and low brightness values on a CT image, and Fig 5 is a graph of brightness using the adaptive threshold method. Fig 6 shows the sequence of the improved segmentation method.

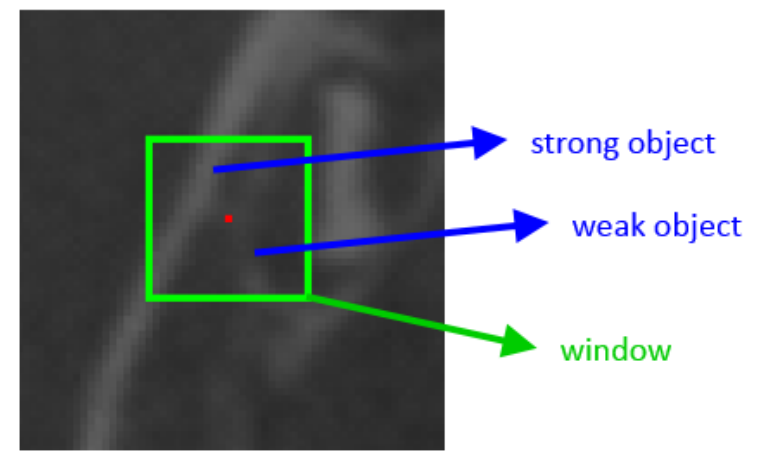

Fig 4 An example of an object with a high brightness value and a low brightness value on a CT image 


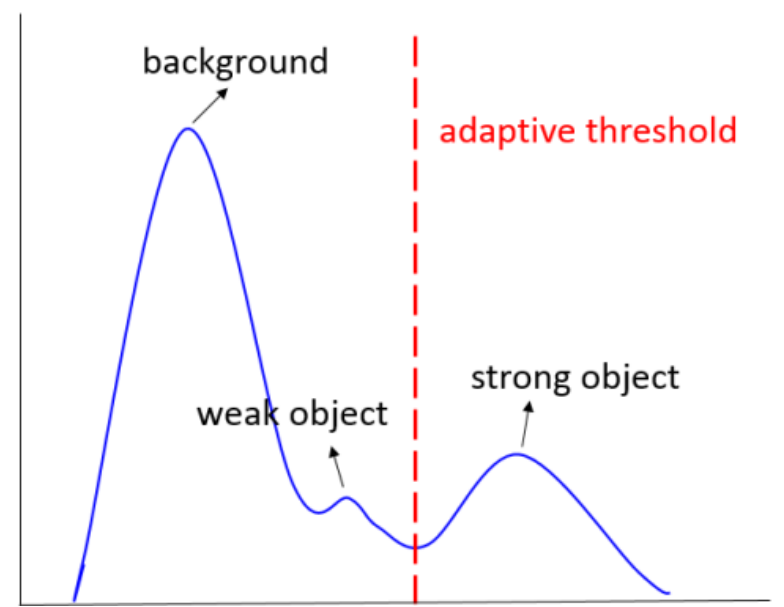

Fig 5 A graph of brightness using the adaptive threshold method

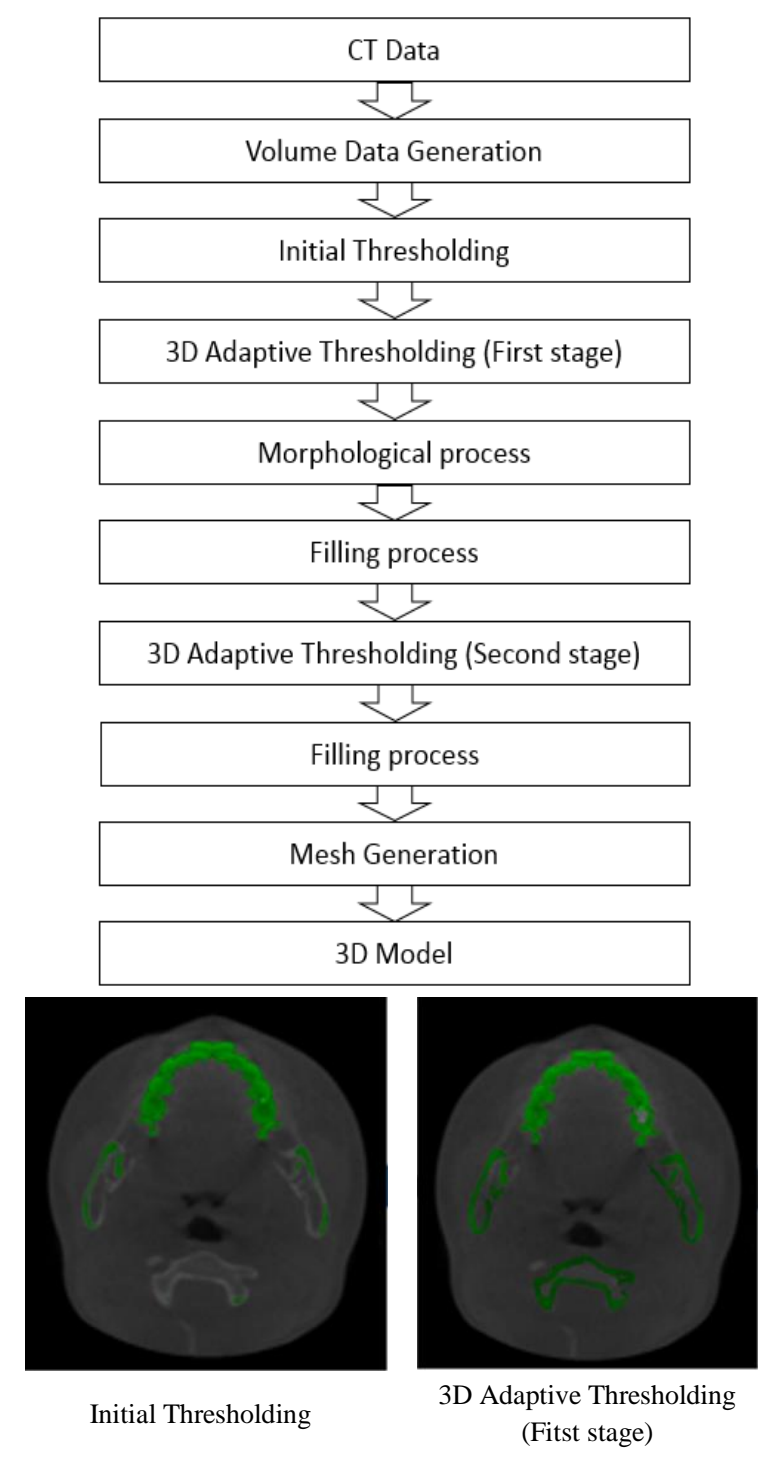

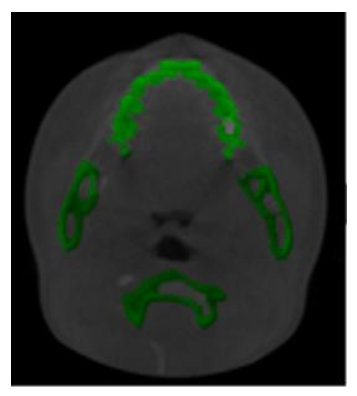

Morphological Process

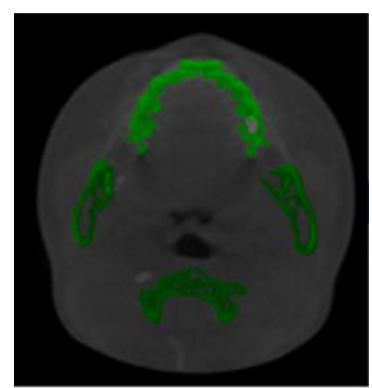

3D Adaptive Thresholding (Second stage)

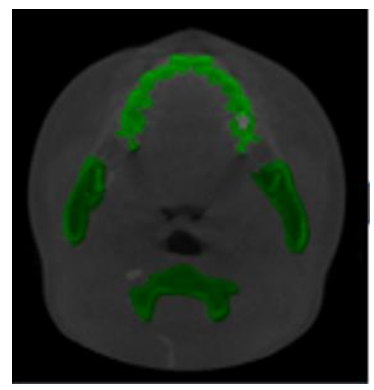

Filling Process

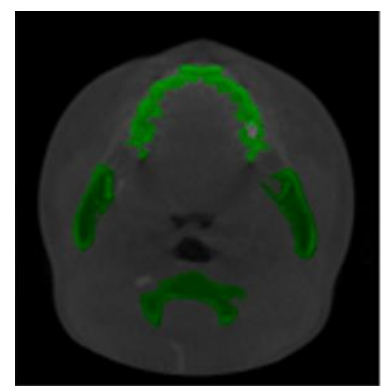

Filling Process

(Final result)
Fig 6 The sequence of the improved segmentation method

\section{Correcting errors in 3D models}

Errors in 3D models are holes, redundant vertices, and inverse normal vectors. The hole error is due to missing triangles in 3D models. This error can occur during the 3D scanning process. Hole error will cause slicing errors, resulting in incorrect 3D printing results. Finding holes in 3D models is solved by finding incomplete edges in triangles. Two triangles must be connected to the edge of a triangle. If there is only one triangle, there is a hole in the edge. When 3D data is large, it takes a long time to compare them as the number of vertices increases. Especially, it takes a very long time to process all the vertices by the sequential search method. To improve this, we used a hash table [7] searching method. We set $\mathrm{x}, \mathrm{y}, \mathrm{z}$ coordinate values as hash key values. The method of finding an incomplete edge using a hash table has been improved with a higher processing speed than using the sequential search method. Fig 7 is an example of hole errors and the result of fixing this error. 

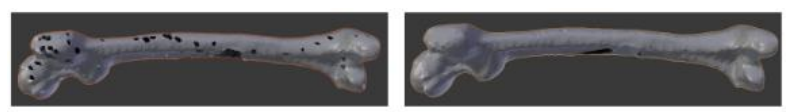

Covaevra
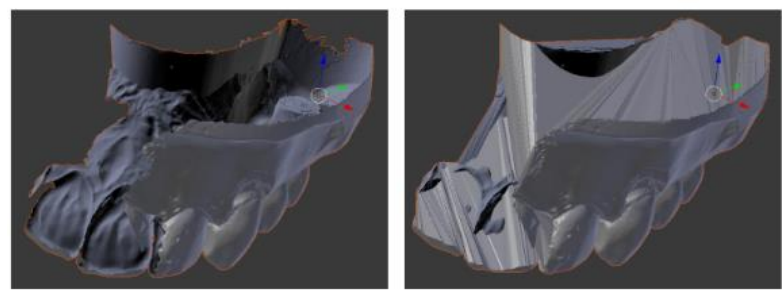

Underjaw

Fig 7 An example of hole errors and the result of fixing this error

\section{Rendering}

A Marching Cube algorithm was used to transform the region of interest of the $\mathrm{CT}$ image into $3 \mathrm{D}$ data [8]. This is an algorithm that makes the isosurface defined in the 3D scalar field (or voxel) a polygon mesh. 3D mesh data can be obtained by extracting the region of interest from CT data and then applying the marching cube algorithm to this data.

OpenGL was used to output 3D models and 2D CT images. The version of OpenGL used in this software was version 1.1 and it has the simple graphics pipeline and coding scheme. To improve the rendering speed, model data was stored and processed in graphics memory through the OpenGL List command.

\section{RESULT AND DISCUSSION}

In order to verify the performance of our improved segmentation method, we compared tooth areas that were separated by the improved segmentation method with the correct answer file that correctly separated the tooth region from the $\mathrm{CT}$ images. The task of accurately separating the tooth region from the CT image was done manually by dental CT imaging specialist doctors at Kyungpook National University. They performed tooth segmentation on $12 \mathrm{CT}$ images containing a core tooth area of the total of $512 \mathrm{CT}$ images. The true positive rate (TPR) was calculated by comparing the images that separated the tooth region using the correct images and the improved segmentation method. The TPR calculates the ratio of how precisely the regions are separated from the entire image. Fig 8 shows the TPR visualizations by comparing the regions of interest using the correct answer images and the improved segmentation method.

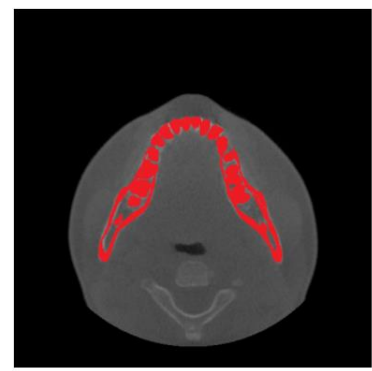

Correct image

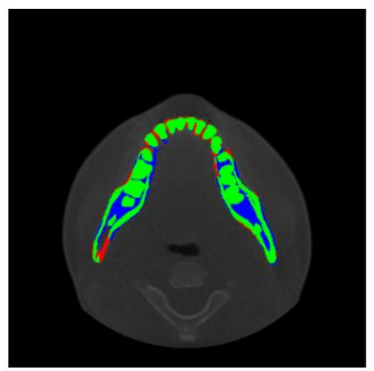

TPR visualization
Fig 8 The TPR visualizations

In the right image of Fig 8, the area shown in greencolored area is the pixel found correctly, and the pixel in red-colored area is the missing pixel. And the bluecolored area is a pixel that should not be found. The average TPR was $88.482445 \%$. Table 1 shows the result of TPR.

Table 1 The result of TPR

\begin{tabular}{|c|c|c|c|}
\hline CT Image & TPR & CT Image & TPR \\
\hline 1 & $89.056686 \%$ & 7 & $89.954544 \%$ \\
\hline 2 & $83.305374 \%$ & 8 & $90.828850 \%$ \\
\hline 3 & $87.226196 \%$ & 9 & $92.267647 \%$ \\
\hline 4 & $93.049049 \%$ & 10 & $87.300049 \%$ \\
\hline 5 & $89.206596 \%$ & 11 & $85.755341 \%$ \\
\hline 6 & $85.466209 \%$ & 12 & $88.372803 \%$ \\
\hline \multicolumn{2}{|c|}{ Average } & $\mathbf{8 8 . 4 8 2 4 4 5} \%$ \\
\hline
\end{tabular}

We calculated the time it took to correct the errors in the 3D model. To improve speed, vertex data is made into a hash table. The method using hash table is improved about from 3 times to 450 times according to the amount of data compared with the sequential search method. Table 2 compares the speed of correcting hole errors using sequential search and hash tables.

Table 2 The speed of correcting hole errors using sequential search and hash tables

\begin{tabular}{|c|c|c|c|}
\hline Model & $\begin{array}{c}\text { Number of } \\
\text { triangles }\end{array}$ & Sequential search & Hash tables \\
\hline coxavera & 13,321 & $3,120 \mathrm{~ms}$ & $912 \mathrm{~ms}$ \\
\hline underjaw & 255,564 & $1,853,120 \mathrm{~ms}$ & $4,213 \mathrm{~ms}$ \\
\hline
\end{tabular}

In $3 \mathrm{D}$ models generated through the marching cube, we added a cropping function to obtain a more detailed model of the region of interest. This is used to remove polygons that are created in some wrongly extracted regions of interest in $3 \mathrm{D}$ models created by our 
improved segmentation method. The direction of cutting the model is perpendicular to the $\mathrm{X}, \mathrm{Y}$, and $\mathrm{Z}$ axes. We used MFC to develop graphical output and GUI functionality. Fig 10 shows the application developed using the methods described above and Fig 11 shows the output using a $3 \mathrm{D}$ printer.

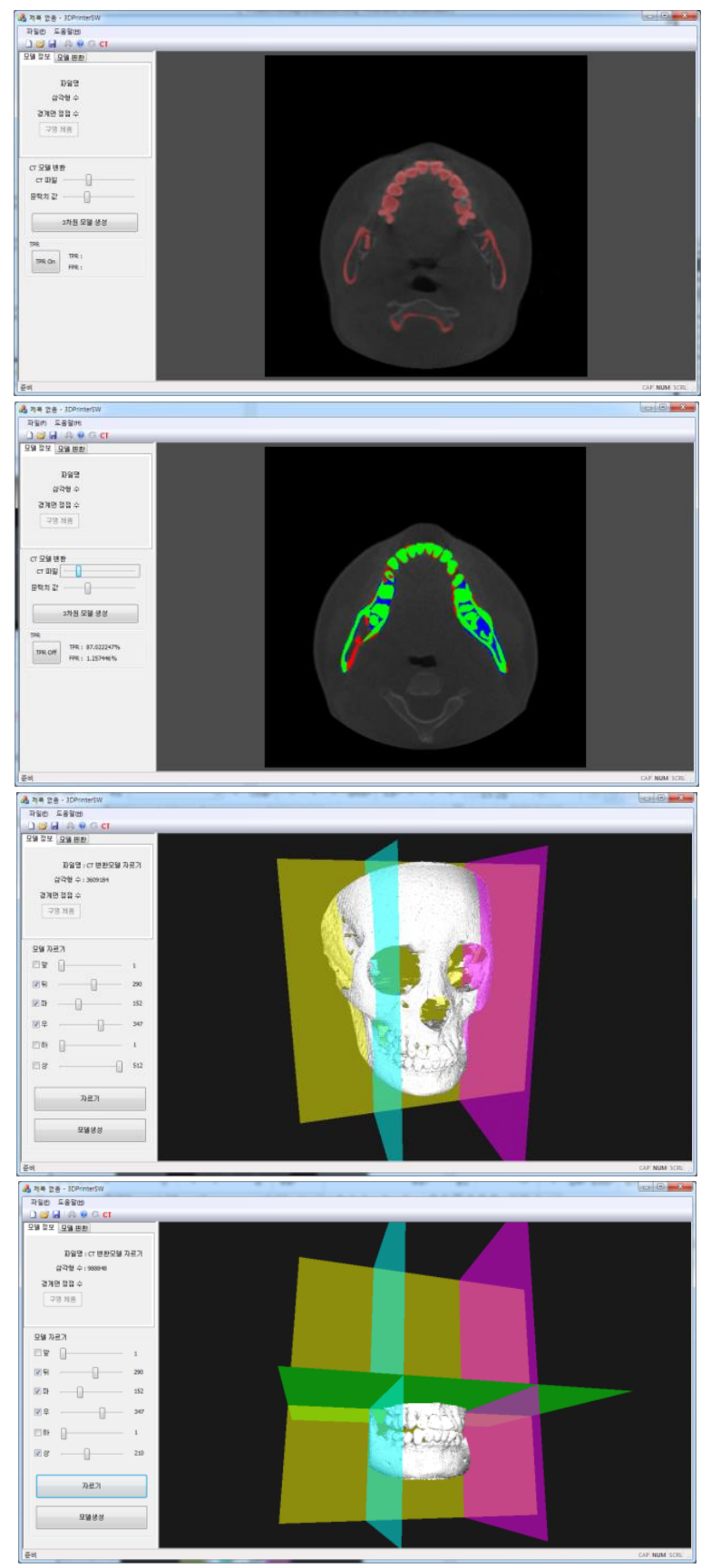

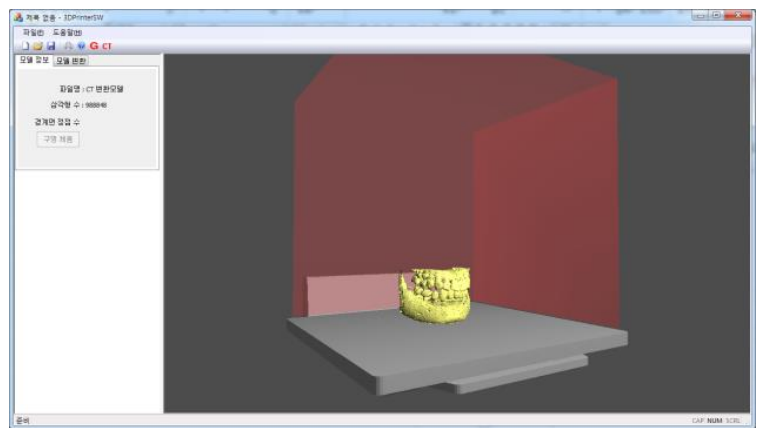

Fig 10 The application

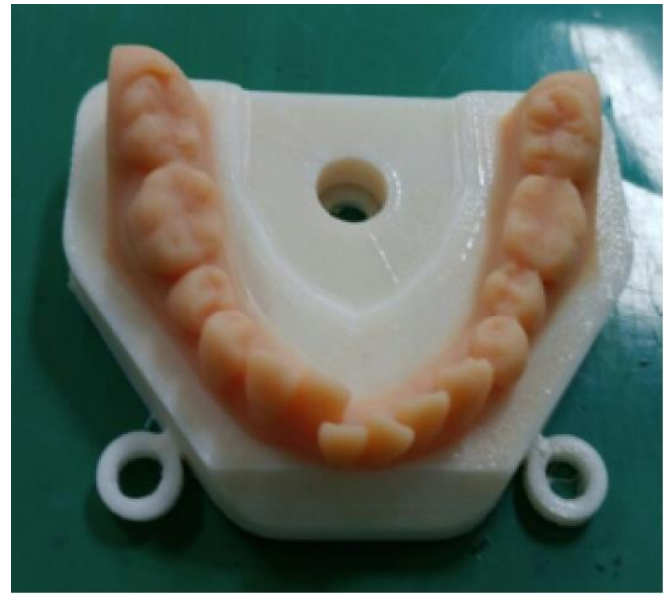

Fig 11 The output using a 3D printer

Such applications can be developed to obtain a desired 3D models in tomographic images and to output 3D models. Although there are applications to obtain 3D models from tomographic images, some areas of interest have been obtained using manual techniques. This research was being planned to automatically extract the area of interest. The improved segmentation method presented in this paper also does not completely separate the region of interest automatically. Therefore, we will perform analysis research on the contrast value of human body part in tomographic images and research on segmentation based on this information. In addition, there is a lack of applications for creating 3D models in tomographic images and supporting both setting and output of 3D printing. It will also be necessary to develop medical 3D printing applications that support a variety of 3D printers suitable for the manufacture of customized treatments.

\section{CONCLUSION}

With the development of 3D printing technology, there is a growing interest in customized treatments. We had 
developed the application that could efficiently extract a region of interest from tomographic images to create 3D model, quickly correct 3D errors of a model, and perform 3D printing. We researched the improved segmentation method to create a 3D model from tomographic images and used a hash table to quickly correct holes and duplicate vertex errors in 3D models. The application also includes the abilities of creating Gcodes visualizing 3D models, cutting 3D models, and supporting 3D printing. The development and advancement of these applications will help to produce customized treatments. In the future, we will research the method of extracting the region of interest automatically from tomographic images, and we will upgrade the application for the manufacture of customized treatments for medical use.

\section{ACKNOWLEDGMENTS}

This work was supported by 2018 collaborative support project using infra-structures of Medical Device Development Center (MDDC), Daegu- Gyeongbuk Medical Innovation Foundation (DGMIF) funded by the Ministry of Trade, Industry \& Energy (MOTIE). (No. 10049767). This work was supported by the ICT R\&D program of IITP/MSIT. (B0101-17-1081, Development of ICT based software platform and service technologies for medical 3D printing applications)

\section{REFERENCES}

[1] Transparency Market Research. 2015. 3DPrinting in Medical Applications Market: Future Trends and Forecast.

[2] 1979. EIA Standard RS-274-D Interchangeable Variable Block Data Format for Positioning, Contouring, and Contouring/Positioning Numerically Controlled Machines. Electronic Industries Association.

[3] M.Szilvasi-Nagy, Gy.Matyasi. 2003. Analysis of STL Files, Mathematical and Computer Modelling 38, 945-960.

[4] D.L.Pham, C.Xu, J.L.Prince. 2000. Current methods in medical image segmentation. Annual Review of Biomedical Engineering2, 315-337

[5] N.Otsu. 1979. A threshold selection method from gray-level histograms. IEEE Trans. Sys., Man., Cyber, 62-66.

[6] Cheolhwan Kim, Jiyoung Yoon, Yun-Jung Lee. 2016. Medical Image Segmentation by More Sensitive Adaptive Thresholding. IEEE Xplore.

[7] Jiyoung Yoon, Wookeun Kim and Mina Lee, A.L. 2017. Hole Finding and Filling in STL File for 3D Printing. Korean Society Precision Engineering Spring.

[8] Lorensen, W. E., Cline, Harvey E. 1987. Marching cubes: A high resolution 3D surface construction algorithm.ACM Computer Graphics 21, 163-169. 